\title{
OPEN Cuticle deposition duration in the uterus is correlated with eggshell cuticle quality in White Leghorn laying hens
}

Xia Chen ${ }^{1,2,4}$, Zhaoxiang $\mathrm{He}^{1,4}$, Xingzheng $\mathrm{Li}^{1,3}$, Jianlou Song ${ }^{1}$, Mingyi Huang ${ }^{1}$, Xuefeng Shi ${ }^{1}$, Xianyu $\mathrm{Li}^{1}$, Junying $\mathrm{Li}^{1}$, Guiyun $\mathrm{Xu}^{1}$ \& Jiangxia Zheng ${ }^{1 凶}$

The cuticle formed in the uterus is the outermost layer as the first defense line of eggshell against microbial invasions in most avian species, and analyzing its genetic regulation and influencing factors are of great importance to egg biosecurity in poultry production worldwide. The current study compared the uterine transcriptome and proteome of laying hens producing eggs with good and poor cuticle quality (GC and PC, the top and tail of the cuticle quality distribution), and identified several genes involved with eggshell cuticle quality (ESCO). Overall, transcriptomic analysis identified 53 differentially expressed genes (DEGs) between PC versus GC group hens, among which 25 were up-regulated and 28 were down-regulated. No differences were found in the uterine proteome. Several DEGs, including PTGDS, PLCG2, ADM and PRLR related to uterine functions and reproductive hormones, were validated by $\mathrm{qPCR}$ analysis. Egg quality measurements between $\mathrm{GC}$ and $\mathrm{PC}$ hens showed $G C$ hens had longer laying interval between two consecutive ovipositions ( $25.64 \pm 1.23$ vs $24.94 \pm 1.12 \mathrm{~h})$ and thicker eggshell thickness $(352.01 \pm 23.04 \mathrm{vs} 316.20 \pm 30.58 \mu \mathrm{m})(P<0.05)$. Apart from eggshell traits, other egg quality traits didn't differ. The result demonstrated eggshell and cuticle deposition duration in the uterus is one of the major factors affecting ESCO in laying hens. PTGDS, $P L C G 2, A D M$ and $P R L R$ genes were discovered and might play crucial roles in cuticle deposition by regulating the uterine muscular activities and secretion function. The findings in the present study provide new insights into the genetic regulation of cuticle deposition in laying hens and establish a foundation for further investigations.

The annual Global Report on Food Crises 2020 by the Food Security Information Network (FSIN) indicates that the world is facing an unprecedented food crisis ${ }^{1}$. As a source of low-cost and high-quality animal protein, poultry production not only meets the basic nutritional needs of humans, but also is an important contributor to the economies and cultures, and thus the demand for poultry egg and meat is increasing ${ }^{2}$. Egg safety is absolutely pivotal to the success of today's poultry industry, however, egg contamination with pathogenic bacteria is considered one of the leading causes of economic loss in the poultry industry worldwide and represents a threat to public health ${ }^{3,4}$. Furthermore, faced with current environmental pressures and animal welfare requirements, the modern poultry industry, which relies on the extensive use of artificial incubation, is more dependent on strict biosecurity of the $\mathrm{egg}^{5}$.

Avian eggshells have evolved multiple physical and chemical barriers in response to microbial challenges, and these barriers are essential for the successful reproduction of avian species as well as to maintain safe and nutritious table eggs for human consumption ${ }^{6,7}$. The eggshell is a complex with several highly ordered and distinct layers (i.e., mammillae, palisades, vertical crystal layer, and cuticle) and the cuticle is the outermost layer as the first defense line of the eggshell against microbial invasions in most avian species ${ }^{8-10}$. The cuticle has been a relatively neglected structure while recent researches on the properties, composition, functions, physiology and genetics of

\footnotetext{
${ }^{1}$ National Engineering Laboratory for Animal Breeding and MOA Key Laboratory of Animal Genetics and Breeding, College of Animal Science and Technology, China Agricultural University, Beijing 100193, China. ${ }^{2}$ Institute of Animal Husbandry and Veterinary Medicine, Beijing Academy of Agriculture and Forestry Sciences, Beijing 100097, China. ${ }^{3}$ Shenzhen Agricultural Genome Research Institute, Chinese Academy of Agriculture Sciences, Shenzhen 440307, China. ${ }^{4} T h e s e$ authors contributed equally: Xia Chen and Zhaoxiang He. ${ }^{\square}$ email: jxzheng@ cau.edu.cn
} 
the cuticle have highlighted its important role in ensuring egg biosecurity and quality in poultry production. The cuticle is deposited during the final hour of egg formation in the uterus (shell gland pouch), covering the shell surface and filling the external openings of the gas pores so that effectively exerting waterproof and antibacterial effects ${ }^{11,12}$. The cuticle mainly consists of hydroxyapatite crystals, glycoprotein, polysaccharides, lipids, and pigment $^{13,14}$, and abundant antibacterial proteins (e.g., lysozyme C, ovotransferrin, ovocalyxin-32, ovocleidin-17) constitute the molecular basis for the antimicrobial function of the cuticle ${ }^{15,16}$. It has been demonstrated that good eggshell cuticle quality (ESCQ) can significantly reduce the opportunity of pathogen invasion ${ }^{5,17,18}$, and the quantity of cuticle is a heritable trait that genetic selection to this trait can be an effective strategy to reduce transmission of microorganisms in poultry production ${ }^{8,19}$. Therefore, how to improve ESCQ to ensure egg biosecurity and quality and further promote poultry production has drawn a worldwide interest.

Avian birds are oviparous and produce an egg at intervals precisely controlled by the hormonal secretion and gene expression of the hypothalamo-pituitary-gonadal-oviduct axis ${ }^{20,21}$. The avian birds' laying cycles are affected by factors such as breeds, age, nutrition, management, physiological status and stress, and the pause day occurs after a laying sequence as a result of cycles in ovulation/oviposition greater than 24 hours $^{22,23}$. Previous transcriptomic and proteomic studies of hen (Gallus gallus) reproductive tract have well profiled the temporal and spatial transcriptome landscapes and important genes that regulate egg formation ${ }^{24-30}$. Cuticle deposition is also a well-defined and specific process that occurs in the uterus rather than the extension of mineralized eggshell ${ }^{12}$. It's reported that there is a significant association between ovocleidin-116, ovocalyxin-32, ovalbumin, and estrogen receptor (ESR1) genes and $\mathrm{ESCQ}^{8,31}$. Transcriptome analysis of the uterus of hens laying eggs with good or poor cuticle quality suggested that clock genes and immediate early genes are prime candidates for the control of cuticle deposition $^{32}$. However, specific pathways and genes that regulate cuticle deposition still remain unclear.

In the present study, transcriptomic and proteomic status of uterus from White Leghorn laying hens that produced eggs with good and poor cuticle quality (GC and PC, the top and tail of the cuticle quality distribution) were analyzed to identify unknown candidate regulatory genes involved in eggshell cuticle deposition and provide deeper insights into the biological basis of cuticle deposition. This is also an accurate study to describe the uterus transcriptome and proteome during the process of chicken eggshell cuticle deposition, providing a reference for further improving the ESCQ in laying hens.

\section{Results}

An overview of GC and PC hens used for transcriptomic and proteomic analysis. Earlier observations of the present study showed that the ESCQ ( $\alpha$ value) may have great variability in the same individual. Therefore, ESCQ of eggs produced by the experimental Flock-A was monitored for the 14 consecutive laying days to obtain individuals that stably produced eggs with good or poor cuticle quality (GC or PC, the top and tail $15 \%$ of the cuticle deposition distribution) for subsequent sampling and analysis. The ESCQ was extremely better in the GC group versus the PC group as indicated by the significant difference in a value (GC $30.18 \pm 7.62$ vs PC $10.34 \pm 3.67$ a value; $\mathrm{n}=7$ and 4 , respectively; F1,9<0.001). In other words, the GC group hens had a strong ability for cuticle deposition whilst the PC groups hens had almost no deposition of cuticle. Such difference in quantity of the cuticle deposition was quite large and suitable for this study. MST cuticle blue dye results of eggs taken from uterus of GC and PC hens during sampling demonstrated that the uterus of GC group had secreted a remarkable thicker cuticle layer than PC group (Supplementary Figure S1). This significant difference of the ESCQ phenotype was in line with expectations that the tissue samples obtained in the present study could be used for subsequent analysis.

Analysis of uterus Transcriptomic data of GC and PC group. Seven and four biological replicate samples of uterus from GC and PC group hens during cuticle formation were analysed. A total of 590,111,516 clean reads were generated from the eleven libraries of GC and PC group (Supplementary Table S1). The reads feature summary indicated that the percentage of reads mapped to Gallus gallus genome was $\geq 85 \%$ (Supplementary Table S2). Differential gene expression analysis showed 53 differentially expressed genes (DEGs) (with 26 genes $\mid \log _{2}$ fold change $>1$ ) between the GC and PC group during cuticle formation. Among the 53 DEGs, there were 25 up-regulated and 28 down-regulated genes at the PC group relative to the GC group. A full list of the 53 DEGs was shown in Table 1.

The heatmap of the 53 DEGs between GC and PC group was plotted and the pattern of expression for the 53 DEGs was visualised in Fig. 1, showing there were significant differences in the expression patterns of the 53 DEGs between GC and PC group.

GO functional annotation and pathway enrichment analysis of the DEGs between GC and PC group. Functional enrichment of Gene Ontology (GO) terms and Kyoto Encyclopedia of Genes and Genomes (KEGG) pathway analysis of the 53 DEGs showed that there was no significant enriched category of GO functional annotation or KEGG pathway when subject to analysis by the DAVID Functional Annotation Tool. This may be due to the small amount of DEGs as only functions of 32 genes from the DEGs are known in the DAVID database.

Verification of gene expression differences by quantitative real-time PCR (qPCR) analysis. To validate the transcriptomics results, the relative expression of four genes [ADM (Adrenomedullin), PLCG2 (Phospholipase C gamma 2), PRLR (Prolactin receptor) and PTGDS (Prostaglandin D2 synthase)] to GAPDH were determined in GC and PC group by qPCR on the basis of their known or potential influence on the hen uterus functions during cuticle deposition. The four genes were all up-regulated in PC group relative to GC group (Table 1). The qPCR results showed a highly similar expression pattern compared with the RNA-Seq 


\begin{tabular}{|c|c|c|c|}
\hline Gene ID & Gene name & padj & $\log _{2}$ FoldChange \\
\hline MSTRG.9297 & LOC112532778 & 0.000107 & -3.38665 \\
\hline MSTRG.4468 & LOC112532092 & 0.012042 & -2.55302 \\
\hline MSTRG.19172 & novel.522 & 0.006783 & -2.46577 \\
\hline MSTRG.14500 & XLOC_018691 & 0.031623 & -2.25265 \\
\hline MSTRG.374243 & WNT2B & 0.006028 & -1.51444 \\
\hline MSTRG.15111 & LOC107054937 & 0.031252 & -1.34327 \\
\hline MSTRG.6687 & XLOC_008333 & 0.041516 & -1.28973 \\
\hline MSTRG.1320 & LOC112530330 & 0.047795 & -1.26421 \\
\hline MSTRG.7221 & LOC112532581 & 0.031623 & -1.2446 \\
\hline MSTRG.10347 & LOC107054090 & 0.005377 & -1.1694 \\
\hline MSTRG.11839 & LOC112533379 & 0.007998 & -1.07028 \\
\hline MSTRG.492 & LOC112533329 & 0.047795 & -0.97969 \\
\hline MSTRG.2992 & ABCB5 & 0.013734 & -0.9499 \\
\hline MSTRG.12238 & XLOC_015835 & 0.00254 & -0.83726 \\
\hline MSTRG.15139 & C1orf167 & 0.019222 & -0.83628 \\
\hline MSTRG.2723 & RNF32 & 0.031623 & -0.59485 \\
\hline MSTRG.12443 & LOC107054608 & 0.030626 & -0.55132 \\
\hline MSTRG.2160 & RCBTB1 & 0.031623 & -0.52116 \\
\hline MSTRG.8764 & LOC112532722 & 0.007866 & $-2.24 \mathrm{E}-05$ \\
\hline MSTRG.8709 & LOC112532731 & 0.041516 & $-1.71 \mathrm{E}-05$ \\
\hline MSTRG.6076 & SLC25A43 & 0.027852 & $-1.66 \mathrm{E}-05$ \\
\hline MSTRG.2635 & OBSCN & 0.041516 & $-1.41 \mathrm{E}-05$ \\
\hline MSTRG.4968 & LOC112532084 & 0.005377 & $-1.26 \mathrm{E}-05$ \\
\hline MSTRG.5952 & LOC112532329 & 0.031623 & $-1.50 \mathrm{E}-06$ \\
\hline MSTRG.12943 & RAD9B & 0.037819 & $-1.31 \mathrm{E}-06$ \\
\hline MSTRG.1636 & LOC112532721 & 0.01454 & $-1.24 \mathrm{E}-06$ \\
\hline MSTRG.8539 & XLOC_011557 & $6.01 \mathrm{E}-09$ & $-2.64 \mathrm{E}-07$ \\
\hline MSTRG.7568 & LOC112532547 & 0.043812 & $7.44 \mathrm{E}-06$ \\
\hline MSTRG.18849 & LOC101747670 & 0.021437 & 0.394261 \\
\hline MSTRG.11340 & PLCG2 & 0.009689 & 0.477929 \\
\hline MSTRG.7243 & $\mathrm{ADM}$ & 0.021437 & 0.660846 \\
\hline MSTRG.2552 & TAF10 & 0.041516 & 0.667103 \\
\hline MSTRG.13459 & PTGDS & 0.006028 & 0.728479 \\
\hline MSTRG.4035 & BAALC & 0.031252 & 0.76145 \\
\hline MSTRG.10742 & BCL2L10 & 0.02381 & 0.805203 \\
\hline MSTRG.430 & LOC101752108 & 0.002939 & 0.866194 \\
\hline MSTRG.17498 & PRLR & 0.000107 & 0.90763 \\
\hline MSTRG.14751 & ID1 & 0.006641 & 0.939962 \\
\hline MSTRG.16737 & TNS4 & 0.049612 & 1.06834 \\
\hline MSTRG.16730 & LOC112530344 & 0.00254 & 1.133301 \\
\hline MSTRG.8092 & XLOC_010789 & 0.009494 & 1.161744 \\
\hline MSTRG.5903 & FGF13 & 0.041516 & 1.167194 \\
\hline MSTRG.4978 & LRP11 & 0.016463 & 1.19355 \\
\hline MSTRG.12672 & LOC101749583 & 0.000618 & 1.21108 \\
\hline MSTRG.8621 & INA & 0.016688 & 1.484377 \\
\hline MSTRG.4238 & LOC107050437 & 0.043077 & 1.577259 \\
\hline MSTRG.11542 & TCTA & 0.000501 & 1.614429 \\
\hline MSTRG.2443 & THRSPB & 0.036456 & 1.677864 \\
\hline MSTRG.2098 & novel.32 & 0.004284 & 1.780021 \\
\hline MSTRG.11804 & novel.277 & 0.007203 & 1.802508 \\
\hline MSTRG.4206 & ARC & 0.036456 & 1.943186 \\
\hline MSTRG.10115 & NEU4 & 0.007866 & 2.080434 \\
\hline MSTRG.5983 & LOC100858332 & 0.000273 & 2.450145 \\
\hline
\end{tabular}

Table 1. DEGs of PC versus GC hen uterus during cuticle deposition from the transcriptomic analysis. 


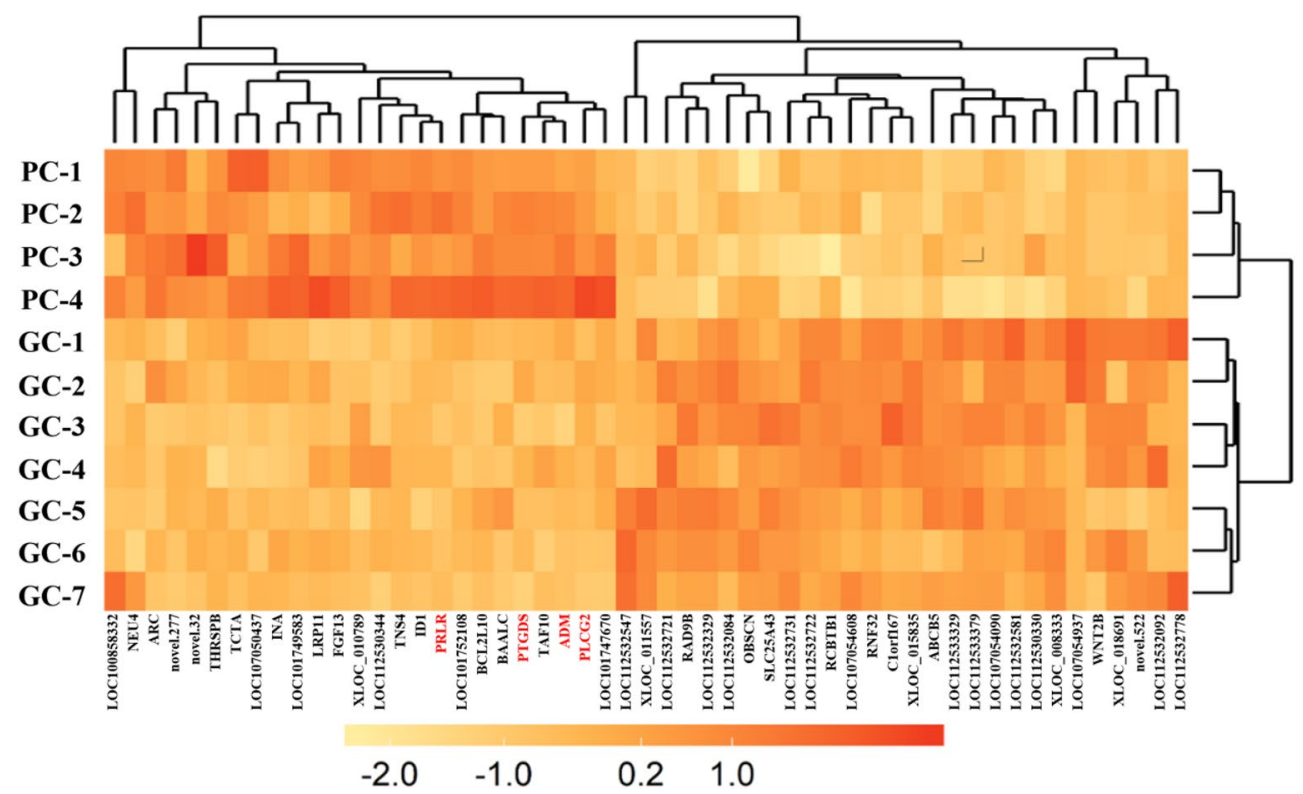

Figure 1. Heatmap of the DEGs between GC and PC hen uterus during cuticle deposition from the transcriptomic analysis. GC, good cuticle; PC, poor cuticle.

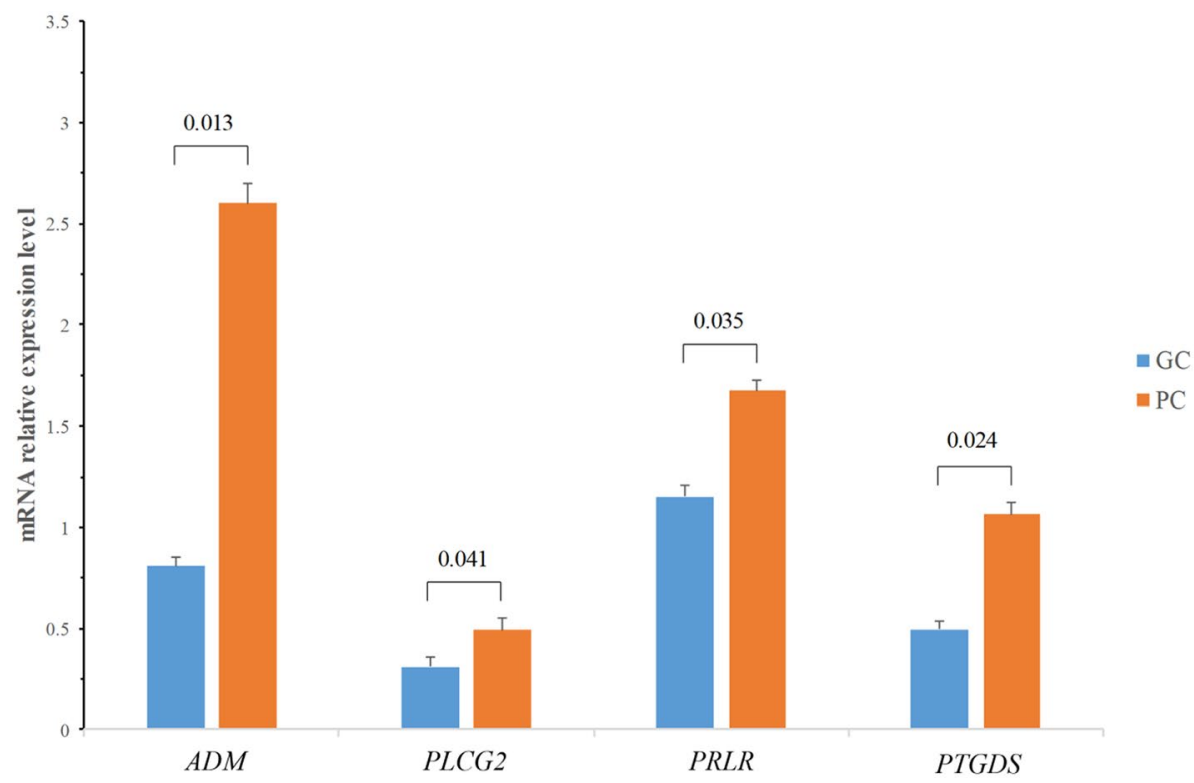

Figure 2. Validation of the DEGs of GC versus PC hen uterus during cuticle deposition from the transcriptomic analysis. The qPCR was performed to quantify the relative gene expression level based on the $2^{-\triangle \Delta \mathrm{CT}}$ method. For gene expression data normalization, GAPDH was used as a reference gene. Fold changes between the GC (good cuticle) and PC (poor cuticle) group were calculated for the genes ADM, PLCG2, PRLR and PTGDS. The Y axis shows the fold changes.

analysis for the genes being validated though there was slight difference in the magnitude of changes in gene expression analyzed by RNA-Seq and qPCR (Fig. 2, Supplementary Table S3). The qPCR results suggested that the RNA-Seq data obtained in the present study provided a good reference for the study of gene expression differences in the uterus of GC and PC group during cuticle deposition.

Proteomic analysis of uterus of GC and PC group. TMT labeling based proteomic analysis of uterus samples of GC and PC group hens ( $n=4$ and 3, respectively) during cuticle formation was further performed to identify differentially expressed proteins (DEPs). The peptide detection results of the TMT sequencing showed 


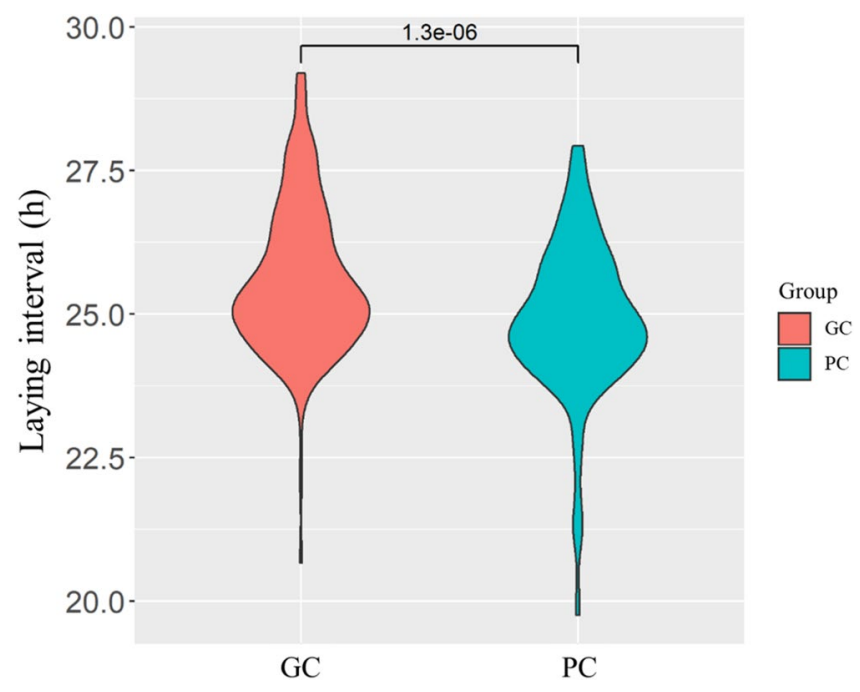

Figure 3. Laying interval of GC and PC group hens. The laying interval between two consecutive ovipositions was determined (at least 4 ovipositions per hen) for GC ( $n=196,60$ hens with 302 ovipositions) and PC ( $\mathrm{n}=180,60$ hens with 280 ovipositions) group hens of Flock-B at 28-week old (GC $25.64 \pm 1.23$ vs PC $24.94 \pm 1.12$, h; $P<0.01)$.

\begin{tabular}{|l|l|l|l|l|}
\hline \multirow{2}{*}{ Traits } & \multicolumn{2}{l}{ Flock-A } & Flock-B \\
\cline { 2 - 5 } & GC & PC & GC & PC \\
\hline $\mathrm{n}^{1}$ & 30 & 30 & 30 & 30 \\
\hline $\mathrm{a}^{2}$ & $33.24 \pm 6.16^{\mathrm{a}}$ & $6.57 \pm 3.00^{\mathrm{b}}$ & $40.26 \pm 5.24^{\mathrm{a}}$ & $13.0 \pm 4.03^{\mathrm{b}}$ \\
\hline Eggshell thickness $(\mu \mathrm{m})^{3}$ & $350.11 \pm 27.75^{\mathrm{a}}$ & $324.49 \pm 35.44^{\mathrm{b}}$ & $368.68 \pm 23.95^{\mathrm{a}}$ & $342.95 \pm 41.28^{\mathrm{b}}$ \\
\hline Eggshell weight $(\mathrm{g})$ & $5.51 \pm 0.50^{\mathrm{a}}$ & $5.10 \pm 0.74^{\mathrm{b}}$ & $5.88 \pm 0.46^{\mathrm{a}}$ & $5.53 \pm 0.75^{\mathrm{b}}$ \\
\hline Eggshell strength $\left(\mathrm{kg} / \mathrm{cm}^{2}\right)$ & $2.87 \pm 0.66^{\mathrm{a}}$ & $2.38 \pm 0.78^{\mathrm{b}}$ & $3.65 \pm 0.78^{\mathrm{a}}$ & $3.02 \pm 0.74^{\mathrm{b}}$ \\
\hline Egg weight $(\mathrm{g})$ & $61.32 \pm 4.46$ & $62.32 \pm 5.51$ & $54.60 \pm 3.62$ & $55.01 \pm 3.10$ \\
\hline Egg yolk weight $(\mathrm{g})$ & $17.82 \pm 1.47$ & $18.43 \pm 1.74$ & $16.07 \pm 3.30$ & $16.60 \pm 1.34$ \\
\hline Egg albumen height $(\mathrm{mm})$ & $6.18 \pm 1.14$ & $6.03 \pm 1.01$ & $6.43 \pm 1.16$ & $6.33 \pm 1.77$ \\
\hline Egg yolk color & $7.91 \pm 0.79$ & $7.85 \pm 1.21$ & $9.32 \pm 0.59$ & $9.31 \pm 0.78$ \\
\hline Haugh unit & $75.67 \pm 7.50$ & $76.23 \pm 11.09$ & $81.15 \pm 7.75$ & $79.22 \pm 12.30$ \\
\hline Egg shape index & $1.36 \pm 0.08$ & $1.35 \pm 0.07$ & $1.35 \pm 0.08$ & $1.35 \pm 0.06$ \\
\hline
\end{tabular}

Table 2. Egg quality between GC and PC eggs. ${ }^{1}$ n, 30 eggs produced by 30 different individuals in each group. ${ }^{2} \alpha$, eggshell cuticle quality (\%). ${ }^{3}$ Eggshell thickness without eggshell membranes. ${ }^{\mathrm{a}, \mathrm{b}}$ Means within a row of the same flock that do not share a common superscript differ significantly $(P<0.05)$.

the length of the peptides is in a partial normal distribution with a mean value of 11 , and the main length is between 8-13, which is reasonable for subsequent proteomic analysis. In the present study, a total of 5177 proteins were identified in the samples and 4296 proteins were quantified (Supplementary Table S4). Proteins with $P<0.01$ by FDR correction were considered as DEPs. Among the DEGs, only PTGDS were expressed at the protein level. Further, the proteomic analysis showed no DEPs were detected between the uterus samples from the GC and PC group hens.

Laying interval, egg quality and laying performance of GC and PC group hens. In order to further verify the results above and explore factors that affect ESCQ, laying interval, egg quality and laying performance were calculated in Flock-A and another White Leghorn laying hen flock (Flock-B) (Supplementary Table S5). Significant differences were found in the laying interval between GC and PC group in both Flock-A and $-\mathrm{B}$ that the GC group hens had a longer laying interval (by about $0.7 \mathrm{~h}$ ) compared to that of PC group $(P<0.05)$ (Fig. 3; Supplementary Table S5). Further, egg quality between GC and PC group hens were measured to investigate possible reasons for the differences in laying interval between the two groups. The results of egg quality indicated that there were significant differences in eggshell thickness (EST), eggshell strength (ESS) and eggshell weight (ESW) between GC and PC group hens $(P<0.05)$, and other egg quality traits were not significantly different, suggesting the differences between GC and PC eggs were mainly reflected in the eggshell qualities (Table 2). Observations and measurements of the eggshell ultrastructure by scanning electron microscope 

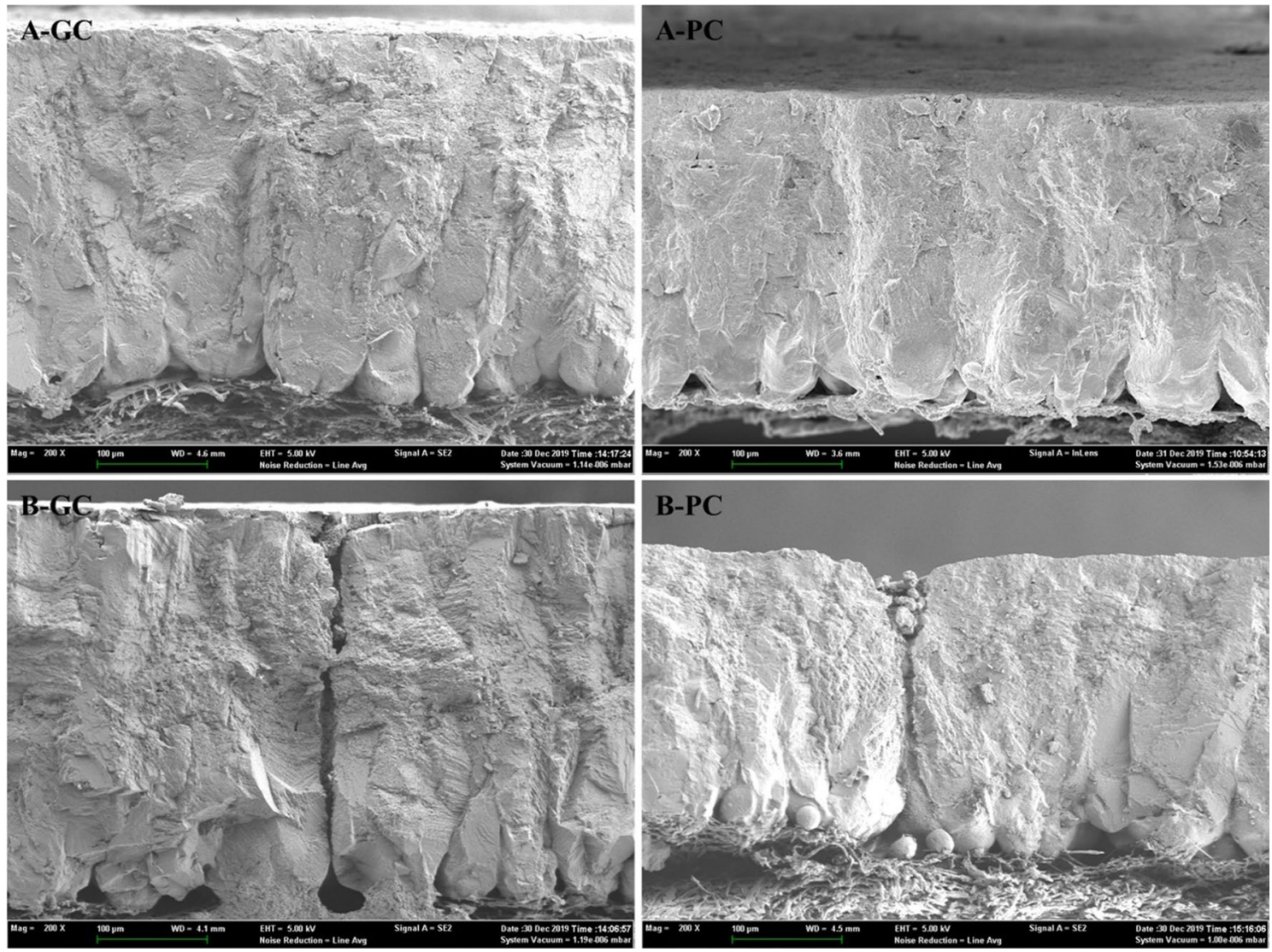

Figure 4. Eggshell ultrastructure of GC and PC group eggs by scanning electron microscope (SEM) $(\times 200)$. A and $\mathrm{B}$, eggshell ultrastructure without and with a gas pore, respectively.

\begin{tabular}{|l|l|l|}
\hline Traits & GC & PC \\
\hline $\mathrm{n}^{1}$ & 30 & 30 \\
\hline $\mathrm{a}^{2}$ & $50.55 \pm 9.53^{\mathrm{a}}$ & $0.29 \pm 2.15^{\mathrm{b}}$ \\
\hline Cuticle thickness $(\mu \mathrm{m})$ & $8.18 \pm 0.83^{\mathrm{a}}$ & $1.52 \pm 0.21^{\mathrm{b}}$ \\
\hline Effective layer thickness $(\mu \mathrm{m})^{3}$ & $281.09 \pm 24.57^{\mathrm{a}}$ & $254.48 \pm 32.18^{\mathrm{b}}$ \\
\hline Mammillary layer thickness $(\mu \mathrm{m})$ & $66.04 \pm 14.97$ & $60.70 \pm 14.11$ \\
\hline Eggshell thickness $(\mu \mathrm{m})$ & $352.01 \pm 23.04^{\mathrm{a}}$ & $316.20 \pm 30.58^{\mathrm{b}}$ \\
\hline
\end{tabular}

Table 3. Eggshell ultrastructure thickness of GC and PC eggs. ${ }^{1} \mathrm{n}, 30$ eggs produced by 30 different individuals in each group of Flock-B. ${ }^{2} \alpha$, eggshell cuticle quality (\%). ${ }^{3}$ Effective layer thickness is the combined thickness of the palisade, vertical crystal and cuticle layer. ${ }^{\mathrm{a}, \mathrm{b}}$ Means within a row that do not share a common superscript differ significantly $(P<0.05)$.

(SEM) also visually showed the huge difference in the cuticle phenotype of GC and PC group hens (Fig. 4). Collectively, the thicker EST of GC group was largely derived from the increase in thickness of the effective layer (including the cuticle layer) $(P<0.05)$ (Fig. 4; Table 3). What's more, Pearson's correlation analysis also showed that the laying interval were positively correlated with the ESCQ (0.31), EST (0.32), ESS (0.33), and ESW (0.33), respectively $(P<0.01)$ (Supplementary Table S6), implying the laying interval has a considerable effect on the eggshell qualities.

\section{Discussion}

Egg formation in avian reproductive tract is strictly regulated by hormones and gene expression ${ }^{24,33}$, and the cuticle layer is the last process of egg formation in most avian species ${ }^{12}$. However, the genetic regulation of cuticle deposition is still poorly understood. The present study was conducted by analyzing the DEGs and DEPs of the uterus between the GC and PC group hens to elucidate potential genes and networks that regulate cuticle deposition. Besides, results of qPCR and a series of phenotypic measurements validated the RNA-Seq data. The current work not only described the differential expression profile of the uterus of GC versus PC group hens during eggshell cuticle deposition, but also revealed important genes may affecting ESCQ. 
Based on the results of DEGs, multiple genes (such as PTGDS, PLCG2, ADM and PRLR) related to uterine functions and reproductive hormones were found. The qPCR results further validated the DEGs that the gene expression levels of PTGDS, PLCG2, ADM and PRLR in the uterus of GC group hens were all lower than those of GC group, suggesting their vital roles during cuticle formation. In mammalian studies, the interaction between these genes and their effect on uterine activity has been well shown. PTGDS is one of the prostaglandin synthases $^{34}$, and it's also an important catalytic enzyme for the synthesis of prostaglandins ${ }^{35,36}$. PLCG2, which is a type of the phospholipase C (PLC), is an important mediator of oxytocin to regulate uterine contractions ${ }^{37}$. Oxytocin increases the synthesis of prostaglandins by stimulating the activity of endometrial prostaglandin synthetase, and then the synergetic effect of prostaglandins and oxytocin enhances the activity of myometrium, thereby inducing parturition in mammals ${ }^{38,39}$. Moreover, ADM could cooperate with oxytocin and prostaglandins to participate in the rhythmical contraction and relaxation of the myometrium ${ }^{40-42}$. The arginine vasopressin and oxytocin are homologous nonapeptides and are known to interact with the other's receptor with different affinities $^{43}$. It is clear that arginine vasopressin and oxytocin arginine vasopressin play an important role in contraction of smooth muscle and parturition in mammals ${ }^{4-46}$. Similar to mammalian arginine vasopressin/oxytocin system, avian arginine vasotocin and mesotocin, which exert 'oxytocic action' inducing uterine muscle contraction during oviposition, are homologous to mammalian arginine vasopressin and oxytocin respectively ${ }^{47-50}$. Argine vasotocin also plays a key role in releasing prostaglandins from the uterus ${ }^{51,52}$. Collectively, under the influence of the hormones argine vasotocin, mesotocin and prostaglandins, uterine muscles contract leading to expulsion of the $\operatorname{~gg}^{52-54}$. Furthermore, arginine vasotocin and prostaglandins is thought to mediate the brain to ovary signalling of oviposition timing being involved in cuticle deposition, and the premature oviposition induced by arginine vasotocin and prostaglandin significantly reduce the EST and ESCQ ${ }^{12}$. Previous study suggested clock gene expression in the uterus during shell formation may be responsible for controlling the cuticle deposition, and clock genes (PER2, CRY2, CRY1, CLOCK and BMAL1) were differentially expressed when cuticle deposition was prevented by arginine vasotocin ${ }^{32}$. Therefore, genes related to uterine timing mechanism and muscular events may constitute the components of cuticle deposition regulation.

Prolactin (PRL), secreted from the anterior pituitary, plays a series of roles in osmoregulation, corpus luteum formation and maintenance of broody behaviour in laying hens, and its receptor, PRLR, plays an important role in the PRL signal transduction cascade and cell growth and differentiation ${ }^{55}$. The PLR and PRLR genes are expressed in many tissues including the hypothalamus, ovary and oviduct ${ }^{56,57}$, and mediate the formation of egg quality ${ }^{58}$. It has been well established that the elevated plasma PRL inhibits gonadotropin release, ovum development and ovulation, resulting poor laying performance and even complete cessation of egg production in laying hens ${ }^{59-61}$. Previous study has also shown the inhibitory effects of excessive PRL on eggshell formation ${ }^{62}$. Besides, it's clear that the elevation of PRL can significantly inhibit the cuticle deposition ${ }^{12,55,63}$. Therefore, the significant difference in eggshell quality (ESCQ, EST, ESS, and ESW) and egg production performance between GC and PC group hens may be related to the different expression patterns of $P R L R$ gene in the uterus. However, the effects of PRL and PRLR on hen uterine function are still unclear.

Summarizing the functions of PLCG2, PTGDS, ADM and PRLR expressed in the uterus, their express patterns in the uterus regulate the muscular activities and secretion function, which may lead to the difference in egg-laying rhythm between GC and PC group hens. The relatively high expressions of PLCG2, PTGDS and ADM genes could increase the frequency and intensity of contraction and relaxation activities of the myometrium that might negatively affect uterine functions and eventually facilitate oviposition accompanied by a reduced duration for the egg staying in the uterus. What's more, our results showed that the laying interval of GC group hens was significantly longer than that of PC group by about $0.7 \mathrm{~h}$. The results above indicated that during the cuticle deposition period in the uterus, the frequency and intensity of myometrium contraction and relaxation in GC group hens might be lower than that of PC group hens, which created a more stable internal environment for the uterus and extend the duration of the cuticle deposition.

However, there were no DEPs between the uterus samples of GC versus PC group hens analyzed by the proteomic analysis, implying there was no significant difference in the protein composition and content to a large extent. Alternative hypothesis is that undetected DEPs may be due to the extensive posttranslational modification regulation in the biological process ${ }^{64,65}$. On the other hand, the extremely short half-life of the hormones may be the reason why the DEPs were not found ${ }^{66-68}$. The expression characteristics of the transcriptome and proteome between GC and PC group are highly similar (53 DEGs and no DEPs), suggesting that the biological processes of cuticle deposition of the two groups may be the same, but mainly the difference in the duration of cuticle deposition. However, since the duration for the uterus to secrete the cuticle is about $1-1.5 \mathrm{~h}$, it was difficult to precisely obtain the uterus samples during cuticle deposition. Though we ensure that the samples and data used in this study are accurate, the relatively small sample size may not be fully representative, and the results still need to be further verified by another study with larger sample size.

From ovulation to oviposition, it takes about $24 \mathrm{~h}$ for the formation of a complete egg in chicken ${ }^{10,33}$. A complete egg includes egg yolk, egg white, eggshell membranes, calcified eggshell and cuticle formation. The forming egg stays in different segments of the oviduct (i.e., infundibulum, magnum, isthmus and uterus) for different duration, and the egg remains in the uterus for the longest period during shell and cuticle formation, for a duration over $18 \mathrm{~h}$ in laying hens ${ }^{10,33,69}$. The egg quality measurement results showed there were no significantly differences in the egg weight, yolk weight, yolk color, albumen height, Haugh unit and egg shape index between GC and PC group. Surprisingly, the eggshell quality (ESCQ, EST, ESS and ESW) of GC group were significantly increased compared with PC group, suggesting that the longer laying interval in GC group was mainly due to the extended formation duration of the eggshell in the uterus. Furthermore, eggshell ultrastructure of GC and PC group eggs by SEM shows the significantly increased EST of GC group was largely due to the increase in the thickness of the effective layer (palisade, vertical crystal layer and cuticle), demonstrating the extended laying interval of GC group hens both positively affects the calcified shell and cuticle deposition. Moreover, the 
laying interval between ovipositions was positively correlated with the duration of eggshell formation, eggshell deposition rate and eggshell quality ${ }^{69}$, which was consistent with the Pearson's correlation results that the laying interval and eggshell quality traits (ESCQ/EST/ESS/ESW) were positively correlated (Supplementary Table S6). Consequently, the prolonged laying interval by $0.7 \mathrm{~h}$ of GC group hens could explain the significant increase in the effective layer and cuticle layer thickness. Therefore, the duration of cuticle deposition in the uterus may be one of the major factors affecting ESCQ. The longer laying interval in GC group hens might be partly derived from the extended cuticle deposition time.

It was notable that the laying interval between oviposition was negatively associated with egg production traits, but it was found that GC group hens with longer laying interval have better egg production performance compared with PC group. What needs to be emphasized is that previous and present studies suggested that there was no negative genetic and phenotypic correlation between ESCQ and production traits ${ }^{19}$. Further, egg production performance are affected by multiple factors such as breeds, nutrition, management and the physiological state of the hen. The increase in egg production by selection largely results from the continuous ovulation of the hen with almost no pause days, which also affects laying interval between oviposition. The PC group hens had shorter mean sequence length and longer inter-sequence pause length, which might be partly associated with the expression patterns of $P L R$ and $P R L R$ genes as discussed above, resulting in relatively worse egg production performance. The White Leghorn laying hens used in the present study was an unselected population about 15 years, while it is remained verification whether the above results consistent with other commercial layer. In a word, though the laying interval of hens with good ESCQ was longer, there was no evidence of any adverse correlation that would prejudice the use of ESCQ as a trait for selection. The ESCQ can be effectively improved and maintained without compromising the egg production with the joint efforts of genetics, nutrition and management. An example is that Hy-Line brown, one of leading egg layer breeds, has the uniform cuticle coverage.

In summary, the physiological state of the uterus regulates the formation of eggshell ultrastructure and quality. By analyzing the transcriptome and proteome of the uterus from hens that produced eggs with good or poor cuticle quality, PTGDS, PLCG2, ADM and PRLR genes were discovered and might play crucial roles in cuticle deposition by affecting uterine secretion rhythm and function. Compared with the PC hens, the relatively low expression of these genes in the uterus ensured the eggshell and cuticle deposition duration, and lead to the good eggshell and cuticle quality of the GC hens.

\section{Methods}

Ethical statement. All the experimental procedures were approved by the Animal Care and Use Committee of China Agricultural University (permit number: AW08059102-1), and all the experiments and animal care protocols were performed in accordance with the Guidelines for Experimental Animals established by the committee. Animal studies were reported in compliance with the ARRIVE guidelines ${ }^{70}$.

Experimental design. One 28-week-old White Leghorn laying hen flocks $(n=208$, Flock-A) was used in present study for omics analysis and then another 28-week-old White Leghorn laying hen flocks ( $\mathrm{n}=574$, FlockB) were chosen for further verification tests. There was no genetic selection for the cuticle trait of the two flocks. Hens that stably produced eggs with good and poor cuticle (GC and PC, the top and tail 15\% of the cuticle deposition distribution) were obtained by evaluating the cuticle quality. According to the ARRIVE guidelines ${ }^{70}$, in the preliminary experiment of the present study, the differences of the top and tail of the cuticle deposition distribution of the two flocks were significant large to obtain enough GC and PC hens for the experiments (GC group hens had a strong ability for cuticle deposition whilst the PC group hens had almost no secretion of cuticle). Transcriptomic, proteomic and qPCR analysis of GC and PC hen uterus tissues during cuticle deposition were designed for identifying genes involved with eggshell cuticle deposition. Subsequently, phenotypic measurements (ESCQ, laying interval, laying performance, serum hormone level, egg quality) of GC and PC group hens were carried out to further verify the results above.

Animals and uterine tissue collection. Two hundred and eight White Leghorn hens at 28-week-old (Flock A) were selected form the National poultry Testing Center, China Agricultural University. All hens were caged $(37.5 \mathrm{~cm}$ length, $40 \mathrm{~cm}$ width and $40 \mathrm{~cm}$ high) individually under standard conditions with a photoperiod of $16 \mathrm{~L}: 8 \mathrm{D}$. The main ingredient of fodder is $16.5 \%$ crude protein, $2.5 \%$ crude fat, $6 \%$ fiber, $13.0 \%$ ash, $2.60-4.00 \%$ calcium, 0.60 phosphorus, $0.20-0.70 \%$ sodium chloride, $0.65 \%$ Met-Cys. The diet and water were provided ad libitum. The temperature and relative humidity of hen house was $22 \pm 1{ }^{\circ} \mathrm{C}$ and $50 \pm 5 \%$, respectively.

To obtain individuals that stably produced eggs with good or poor cuticle (GC or PC) for subsequent sampling, the eggs were collected for 14 consecutive days to measure the ESCQ within $24 \mathrm{~h}$ after oviposition. The ESCQ evaluation was conducted according to the staining method proposed by Chen et al. previously ${ }^{17}$. Briefly, the ESCQ ( $a$ value) was measured based on differences in cuticle staining before and after staining with a dye solution of MST cuticle blue (MS Technologies Ltd, UK) using a spectrophotometer (CM-2600d; Konica Minolta, Japan) with the XYZ color space system. A higher a value represents to more cuticle deposition, that is better ESCQ. Each egg was measured at 3 points: the large end, equator, and small end. ESCQ per egg was determined from the mean value of these points and at least 8 eggs were collected for ESCQ measurement per hen.

Oviposition time of each hen was recorded from the eighth to fourteen day of egg collection. The oviposition time was manually recorded every 5-10 $\mathrm{min}$, from 5:30 to 22:00 every day. The experimenters were trained to minimize interference with the hens. Then the oviposition time of the GC and PC group hens were estimated and the hens were euthanized by T-61 intravenously $(0.4 \mathrm{ml} / \mathrm{kg}) 1 \mathrm{~h}$ before oviposition (the period for cuticle secretion) on the fifteenth day. The uterus was aseptically retracted through an abdominal incision and a small incision was then made in the centre of the uterus, and the egg in the uterus was removed for staining by MST 
cuticle blue within 30 min to determine whether the cuticle is deposited in the uterus during sampling. Two approximately $500 \mathrm{mg}$ sample tissues was collected from the centre of the uterus and transferred directly to RNAlater (Sigma-Aldrich, Shanghai, China), quickly frozen in liquid nitrogen and stored at $-85^{\circ} \mathrm{C}$ prior to total RNA extraction.

RNA isolation, library construction, and sequencing. Total RNA was extracted from frozen uterus tissue sample using TRIzol reagent (TransGen Biotech, Beijing, China) ${ }^{71}$. The quality and quantity of RNA were evaluated by $1 \%$ agarose gels, NanoDrop 2000 spectrophotometer (Thermo Fisher Scientific, Wilmington, DE, USA), respectively. The RNA integrity was assessed using the RNA Nano 6000 Assay Kit of the Bioanalyzer 2100 system (Agilent Technologies, CA, USA). $3 \mu \mathrm{g}$ RNA per sample was prepared to generate individual barcoded sequencing libraries using NEBNext ${ }^{\circledR}$ UltraTM RNA Library Prep Kit for Illumina ${ }^{\circledR}$ (NEB, USA) following manufacturer's recommendations. Sequencing of these libraries was performed on an Illumina HiSeq 4000 sequencing system (Illumina, San Diego, CA, USA) using the 150-bp pair-end sequencing strategy, following the manufacturer's instructions.

Transcriptomic data analysis. Raw data was quality controlled using the FastQC package (Babraham bioinformatics, Cambridgeshire, England). Clean reads were obtained by removing reads containing adapter, reads containing poly- $\mathrm{N}$, empty reads and low quality reads from raw data. Trimmed reads were mapped on the reference chicken genome Gallus_gallus_ncbi_GCF_000002315.6_GRCg6a (https://www.ncbi.nlm.nih.gov/ assembly/GCF_000002315.6) using HiSAT2.072,73. The clean reads of each sample were assembled and finally merged to a transcriptome using Stringtie (http://ccb.jhu.edu/software/stringtie/) with Ensembl Gallus gallus. v92 as the reference ${ }^{72}$. Expression levels of the transcripts were quantified as RPKM (reads per kilobase per million) for gene expression analysis.

DEGs were identified using DESeq 2 according to the cretiera of adjusted $P$-value $<0.05^{74}$. Ensembl gene IDs from each group were uploaded to the DAVID Functional Annotation Tool and analysed for gene ontology (GO) and Kyoto Encyclopaedia of Genes and Genomes (KEGG) enrichment (https://www.kegg.jp/kegg/pathw ay.html). ${ }^{75-77}$ The transcriptome data (raw mRNA-seq reads) have been deposited with the National Center for Biotechnology Information (NCBI) Sequence Read Archive (SRA) database (https://www.ncbi.nlm.nih.gov/ sra/PRJNA664894).

Sample processing and liquid chromatography coupled with tandem mass spectrometry (LCMS/MS). Each frozen sample (50 mg) from GC and PC group hens ( $\mathrm{n}=4$ and 3, respectively) was ground in liquid nitrogen and suspended in lysis buffer consisting of $8 \mathrm{M}$ urea (Sigma Aldrich, MO, USA), $10 \mathrm{mM}$ dithiothreitol (DTT), and proteinase inhibitors (Merck Millipore, MA, USA). The suspension was centrifuged at $12,000 \times \mathrm{g}$ for $20 \mathrm{~min}$ at $4{ }^{\circ} \mathrm{C}$, and the supernatant was collected. The concentration was determined with a BCA kit (Thermo Fisher Scientific, Wilmington, DE, USA) according to the manufacturer's protocols. After trypsin digestion, the peptides were desalted with buffer A $\left[10 \mathrm{mM} \mathrm{KH}_{2} \mathrm{PO}_{4}\right.$ in $25 \%$ acetonitrile $(\mathrm{ACN}), \mathrm{pH}$ 3.0] and buffer $\mathrm{B}\left(10 \mathrm{mM} \mathrm{KH}_{2} \mathrm{PO}_{4}, 500 \mathrm{mM} \mathrm{KCl}\right.$, in $\left.25 \% \mathrm{ACN}, \mathrm{pH} 3.0\right)$ at a low rate of $1,000 \mu \mathrm{L} / \mathrm{min}$, lyophilised in a centrifugal speed vacuum concentrator, reconstituted in $0.5 \mathrm{M} \mathrm{TEAB}$ and processed according to the manufacturer's instructions for the TMT kit (Thermo Fischer Scientific, MA, USA). The tryptic peptides were separated by high-pH reverse-phase HPLC using an Agilent 300 Extend C 18 column (5 $\mu$ m particle size, $4.6 \mathrm{~mm}$ inner diameter, $250 \mathrm{~mm}$ length), dissolved in $0.1 \%$ formic acid (Sigma Aldrich, MO, USA) and fractionated using EASY-Nano-LC 1000 ultra-high performance liquid phase system. Finally, fractionated peptides were exposed to an NSI source followed by tandem mass spectrometry (MS/MS) with a Q Exactive HF-X spectrometer (Thermo Fischer Scientific, MA, USA) coupled online to the UPLC system.

The MS/MS data were searched against the Gallus gallus database (http://www.uniprot.org/proteomes/UP000 000539, chicken proteome ID: UP000000539) for peptide identification and quantification using the Maxquant search engine (v.1.6.6.0). All proteins with at least one unique peptide and false discovery rate (FDR) $<0.01$ were qualified for further quantification data analysis. Protein abundance was quantified by hi-flyer through loading (SL), internal reference scaling (IRS) and trimmed mean of M values (TMM) corrections in the Maxquant search engine system. DEPs between GC and PC group were identified using limma and $P<0.05$ was used as the cut-off for significance by FDR correction ${ }^{78}$.

Quantitative real-time PCR (qPCR) analysis. Expression of mRNA was verified by qPCR with cDNA from 11 uterine tissue samples. GAPDH gene served as a housekeeping gene. Primer Primer (v. 5.0) was used with default parameters to design primers on exon-exon spans for selected genes (Supplementary Table S7). Total RNA was extracted from uterine tissue using TRIzol reagent as described previously. Synthesis of the cDNA was performed using a PrimeScript RT reagent kit with $1 \mu \mathrm{g}$ of the RNA pretreated with gDNA Eraser (TaKaRa, Dalian, China) following the manufacturer's instructions. The qPCR of the mRNA expression level of ADM, PLCG2, PRLR and PTGDS was performed using the Agilent Brilliant III Ultra-Fast SYBR Green qPCR Mix and MX3005P real time system (Agilent; Santa Clara, CA USA). The experiments were carried out in triplicate. The cycling conditions were $50^{\circ} \mathrm{C}$ for $2 \mathrm{~min}$, followed by 40 cycles at $95^{\circ} \mathrm{C}$ for $15 \mathrm{~s}$ and $60^{\circ} \mathrm{C}$ for $1 \mathrm{~min}$ by 42 cycles, and annealing and extension at $60^{\circ} \mathrm{C}$ for $15 \mathrm{~s}$. The melting curves were obtained for each sample amplified. The $2^{-\triangle \triangle C T}$ method was used to quantify the relative changes in gene expression versus those of GAPDH from the qPCR experiments ${ }^{79}$, and $t$-test was used for the statistical analysis by R Language (v. 3.4.0).

Phenotypic measurements and experimental details. After the ESCQ measurement, the eggs produced by the GC and PC hens $(n=30)$ of Flock-A above were randomly selected for the phenotypic measure- 
ments of egg quality traits. Egg weight, eggshell thickness (EST, mean of the large end, equator, and small end without eggshell membranes), eggshell strength (ESS), eggshell weight (ESW), yolk weight, albumen height, yolk colour, Haugh unit and egg shape index (ESI) were determined using an egg multitester (EMT-5200, robotmation, Japan), an Eggshell Strength Tester (EFG-0503, robotmation, Japan) and a digital display micrometer gauge (Mitutoyo, Kawasaki, Japan) following the manufacturer's instructions. Laying performance (i.e., total laying days, total sequences, mean sequence length and intersequence pause length) was calculated by the 14-days egg collection. Laying interval between two consecutive ovipositions was calculated and the laying interval of each group was expressed by the average (at least 4 ovipositions per hen).

Further, Five hundred and seventy four White Leghorn hens at 28-week-old (Flock-B) were selected form the Poultry Genetic Resources and Breeding Experimental Base, College of Animal Science and Technology, China Agricultural University for subsequent verification analysis. All hens were keep in individually cage $(37.5 \mathrm{~cm}$ length, $23.5 \mathrm{~cm}$ width and $40 \mathrm{~cm}$ high) and feeding with the same fodder and photoperiod as described above, and the diet and water were provided ad libitum. The temperature and relative humidity of hen house is $22 \pm 1$ ${ }^{\circ} \mathrm{C}$ and $50 \pm 5 \%$ controlled by an automated system, respectively.

All eggs produced by Flock-B were collected, and ESCQ measurement and oviposition time recording were the same as described above for seven successive laying days. Laying recording sheet during the 27 consecutive laying days from 28-week-old age was obtained to evaluate the laying performance. The laying interval and egg quality traits of GC and PC group hens $(n=30)$ were measured as described above. After the egg quality measurements, eggshell pieces (about $1 \mathrm{~cm}^{2}$ ) of eggs above from the GC and PC group hens $(\mathrm{n}=30)$ were taken around the equator of the egg and the pieces were mounted on an aluminum stub and gold sputter-coated using an EIKO IB-3 (EIKO Engineering CO., Ltd, Japan) for about $15 \mathrm{~min}$. Thereafter, they were subjected to the SEM (JSM-7401, JEOL Ltd., Japan) for observations, photographing, and measurements of the eggshell cross-section ultrastructure. The eggshell cross-section ultrastructure in the present study refers to EST, effective layer thickness (combined thickness of the palisade, vertical crystal and cuticle layer) and mammillary layer thickness, and the determination were consistent with previous studies ${ }^{31,80}$.

Except for the GC and PC eggs, other eggs collected from Flock-A and Flock-B during the experiments were further measured for egg quality, respectively (at least one egg was measured per hen).

Statistical analysis. Statistically significant differences among descriptive statistics were determined by the Student's $t$ test. The phenotypic correlation between the egg quality and laying performance traits was estimated by Pearson's correlation coefficient. All statistical analysis and figure plotting were performed with the statistical software RStudio (v. 3.4.0). The results of basic descriptive statistics are shown in paragraphs and tables using the mean and standard deviation (mean $\pm \mathrm{SD})$.

Received: 18 May 2021; Accepted: 1 November 2021

Published online: 11 November 2021

\section{References}

1. FSIN. The Global Report on Food Crises 2020. https://www.fsinplatform.org/sites/default/files/resources/files/GRFC_2020_ ONLINE_200420.pdf (2020).

2. Mottet, A. \& Tempio, G. Global poultry production: Current state and future outlook and challenges. World's Poult. Sci. J. 73, 1-12 (2017).

3. Gantois, I. et al. Mechanisms of egg contamination by Salmonella Enteritidis: Review article. FEMS Microbiol. Rev. 33, 718-738 (2009).

4. Baron, F., Jan, S., Nys, Y., Bain, M. \& Immerseel, F. Egg and egg product microbiology. Improving the safety and quality of eggs and egg products Vol 1: Egg chemistry, production and consumption 330-350 (Woodhead Publishing, Cambridge, 2011).

5. Bain, M. et al. Cuticle deposition improves the biosecurity of eggs through the laying cycle and can be measured on hatching eggs without compromising embryonic development. Poult. Sci. 98, 1775-1784 (2018).

6. D'Alba, L. \& Shawkey, M. Mechanisms of antimicrobial defense in avian eggs. J. Ornithol. 156, 399-408 (2015).

7. Hincke, M. et al. The eggshell: Structure and protective function. In Improving the Safety and Quality of Eggs and Egg Products Volume 1: Egg Chemistry, Production and Consumption (eds Nys, Y. et al.) 151-182 (Woodhead Publishing, Cambridge, 2011).

8. Bain, M. et al. Enhancing the egg's natural defence against bacterial penetration by increasing cuticle deposition. Anim. Genet. 44, 661-668 (2013).

9. D’Alba, L., Jones, D., Eliason, C., Badawy, H. \& Shawkey, M. Antimicrobial properties of a nanostructured eggshell from a compostnesting bird. J. Exp. Biol. 217, 1116-1121 (2014).

10. Hincke, M. et al. The eggshell: Structure, composition and mineralization. Front Biosci. 17, 1266-1280 (2012).

11. Kulshreshtha, G., Rodrigueznavarro, A., Sanchezrodriguez, E., Diep, T. \& Hincke, M. T. Cuticle and pore plug properties in the table egg. Poult. Sci. 97, 1382-1390 (2018).

12. Wilson, P. et al. Understanding avian egg cuticle formation in the oviduct; a study of its origin and deposition. Biol. Reprod. 97 , 39-49 (2017).

13. Kusuda, S., Iwasawa, A., Doi, O., Ohya, Y. \& Yoshizaki, N. Diversity of the cuticle layer of avian eggshells. J. Poult. Sci. 48, 119-124 (2011).

14. Fecheyr-Lippens, D. C. et al. The cuticle modulates ultraviolet reflectance of avian eggshells. Biol. Open. 4, 753-759 (2015).

15. Wellman-Labadie, O., Picman, J. \& Hincke, M. T. Antimicrobial activity of the Anseriform outer eggshell and cuticle. Comp. Biochem. Physiol. 149, 640-649 (2008).

16. Rose-Martel, M., Du, J. \& Hincke, M. T. Proteomic analysis provides new insight into the chicken eggshell cuticle. J. Proteomics 75, 2697-2706 (2012).

17. Chen, X. et al. Impact of cuticle quality and eggshell thickness on egg antibacterial efficiency. Poult. Sci. 98, 940-948 (2018).

18. Chen, X. et al. Comparative study of eggshell antibacterial effectivity in precocial and altricial birds using Escherichia coli. PLoS ONE 14, e220054 (2019). 
19. Dunn, I. et al. Genetic variation and potential for genetic improvement of cuticle deposition on chicken eggs. Genet. Sel. Evol. 51, $1-13(2019)$.

20. Etches, R. J. \& Cunningham, F. J. The interrelationship between progesterone and luteinizing hormone during the ovulation cycle of the hen (Gallus domesticus). J. Endocrinol. 71, 51-58 (1976).

21. Wilson, S. C. \& Sharp, P. J. Changes in plasma concentrations of luteinizing hormone after injection of progesterone at various times during the ovulatory cycle of the domestic hen (Gallus domesticus). J. Endocrinol. 67, 59-70 (1975).

22. Mills, A. D., Nys, Y., Gautron, J. \& Zawadski, J. Whitening of brown shelled eggs: Individual variation and relationships with age, fearfulness, oviposition interval and stress. Br. Poult. Sci. 32, 117-129 (1991).

23. Reddy, I. J., David, C. G., Selvaraju, S., Mondal, S. \& Ravi, K. G. GnRH-1 mRNA, LH surges, steroid hormones, egg production, and intersequence pause days alter in birds exposed to longer wavelength of light in the later stages of production in Gallus gallus domesticus. Trop Anim Health Prod. 44, 1311-1317 (2012).

24. Yin, Z. et al. The transcriptome landscapes of ovary and three oviduct segments during chicken (Gallus gallus) egg formation. Genomics 112, 1-9 (2020).

25. Marie, P. et al. Quantitative proteomics and bioinformatic analysis provide new insight into protein function during avian eggshell biomineralization. J. Proteomics. 113, 178-193 (2014).

26. Sun, C., Xu, G. \& Yang, N. Differential label-free quantitative proteomic analysis of avian eggshell matrix and uterine fluid proteins associated with eggshell mechanical property. Proteomics 13, 23-24 (2013).

27. Jonchere, V. et al. Gene expression profiling to identify eggshell proteins involved in physical defense of the chicken egg. BMC Genom. 11, 57 (2010).

28. Brionne, A., Nys, Y., Hennequet-Antier, C. \& Gautron, J. Hen uterine gene expression profiling during eggshell formation reveals putative proteins involved in the supply of minerals or in the shell mineralization process. BMC Genom. 15, 220 (2014).

29. Du, J. et al. Identifying specific proteins involved in eggshell membrane formation using gene expression analysis and bioinformatics. BMC Genom. 16, 792 (2015).

30. Khan, S., Wu, S. \& Roberts, J. RNA-sequencing analysis of shell gland shows differences in gene expression profile at two timepoints of eggshell formation in laying chickens. BMC Genom. 20, 89 (2019).

31. Dunn, I. et al. Polymorphisms in eggshell organic matrix genes are associated with eggshell quality measurement in pedigree Rhode Island Red hens. Anim. Genet. 40, 110-114 (2009).

32. Pertiñez, S. et al. Transcriptome analysis of the uterus of hens laying eggs differing in cuticle deposition. BMC Genom. 21, 516 (2020).

33. Sah, N. \& Mishra, B. Regulation of egg formation in the oviduct of laying hen. World's Poult. Sci. J. 74, 1-13 (2018).

34. Kanaoka, Y. et al. Cloning and crystal structure of hematopoietic prostaglandin D synthase. Cell 90, 1085-1095 (1997).

35. Urade, Y. \& Eguchi, N. Lipocalin-type and hematopoietic prostaglandin D synthases as a novel example of functional convergence. Prostag. Oth. Lipid M. 68-69, 375-382 (2002).

36. Gross, G., Imamura, T. \& Muglia, L. J. Gene knockout mice in the study of parturition. J. Soc. Gynecol. Invest. 7, 88-95 (2000)

37. Phaneuf, S., Carrasco, M. P., Europe-Finner, G. N., Hamilton, C. H. \& López Bernal, A. Multiple G proteins and phospholipase C isoforms in human myometrial cells: Implication for oxytocin action. J. Clin. Endocrinol. Metab. 81, 2098-2103 (1996).

38. Phillippe, $M$. The relationship between oxytocin, phosphoinositide-specific phospholipase $\mathrm{C}$, and phasic myometrial contractions. J. Soc. Gynecol. Invest. 1, 49-54 (1994).

39. Ku, C. Y., Qian, A., Wen, Y., Anwer, K. \& Sanborn, B. M. Oxytocin stimulates myometrial guanosine triphosphatase and phospholipase-C activities via coupling to G alpha q/11. Endocrinology 136, 1509-1515 (1995).

40. Salomonis, N. et al. Identifying genetic networks underlying myometrial transition to labor. Genome Biol. 6, R12 (2005).

41. Thota, C. \& Yallampalli, C. Progesterone upregulates calcitonin gene-related peptide and adrenomedullin receptor components and cyclic adenosine $3^{\prime} 5^{\prime}$-monophosphate generation in eker rat uterine smooth muscle cell line. Biol. Reprod. 72, 416-422 (2005).

42. Norwitz, E. R., Robinson, J. N. \& Challis, J. R. The control of labor. New Engl. J. Med. 341, 660-666 (1999).

43. Akerlund, M. et al. Receptor binding of oxytocin and vasopressin antagonists and inhibitory effects on isolated myometrium from preterm and term pregnant women. Br. J. Obstet. Gynaecol. 106, 1047-1053 (1999).

44. Bossmar, T. et al. Receptors for and myometrial responses to oxytocin and vasopressin in preterm and term human pregnancy: Effects of the oxytocin antagonist atosiban. Am. J. Obstet. Gynecol. 171, 1634-1642 (1994).

45. Gimpl, G. \& Fahrenholz, F. The oxytocin receptor system: Structure, function, and regulation. Physiol. Rev. 81, 629-683 (2001).

46. Thornton, S. et al. The role of arginine vasopressin in human labour: Functional studies, fetal production and localisation of V1a receptor mRNA. BJOG 109, 57-62 (2002).

47. Sawyer, W. H. Evolution of active neurohypophysial principles among the vertebrates. Am. Zool. 17, 727-737 (1977).

48. Koike, T. I., Shimada, K. \& Cornett, L. E. Plasma levels of immunoreactive mesotocin and vasotocin during oviposition in chickens: Relationship to oxytocic action of the peptides in vitro and peptide interaction with myometrial membrane binding sites. Gen. Comp. Endocrinol. 70, 119-126 (1988).

49. Takahashi, T. \& Kawashima, M. Mesotocin increases the sensitivity of the hen oviduct uterus to arginine vasotocin. Poult. Sci. 87, 2107-2111 (2008).

50. Wu, C. et al. Arginine vasotocin (AVT)/mesotocin (MT) receptors in chickens: Evidence for the possible involvement of AVTAVPR1 signaling in the regulation of oviposition and pituitary prolactin expression. Gen. Comp. Endocrinol. 281, 91-104 (2019).

51. Rzasa, J. The effect of arginine vasotocin on prostaglandin production of the hen uterus. Gen. Comp. Endocrinol. 53, 260-263 (1984).

52. Saito, N., Shimada, K. \& Koike, T. I. Interrelationship between arginine vasotocin, prostaglandin, and uterine contractility in the control of oviposition in the hen (Gallus domesticus). Gen. Comp. Endocrinol. 67, 342-347 (1987).

53. Hertelendy, F., Yeh, M. \& Biellier, H. V. Induction of oviposition in the domestic hen by prostaglandins. Gen. Comp. Endocrinol. 22, 529-531 (1974).

54. Sasaki, T., Shimada, K. \& Saito, N. Changes of AVT levels in plasma, neurohypophysis and hypothalamus in relation to oviposition in the laying hen. Comp. Biochem. Physiol. A Mol. Integr. Physiol. 121, 149-153 (1998).

55. Wilkanowska, A., Mazurowski, A., Mroczkowski, S. \& Kokoszynski, D. Prolactin (PRL) and prolactin receptor (PRLR) genes and their role in poultry production traits. Folia. Biol. 62, 1-8 (2014).

56. Ohkubo, T., Tanaka, M. \& Nakashima, K. Molecular cloning of the chicken prolactin gene and activation by Pit-1 and cAMPinduced factor in GH3 cells. Gen. Comp. Endocrinol. 119, 208-216 (2000).

57. Xing, G., Zhao, Q., Mao, J., Liu, T. \& Wang, G. Identification and characterization of goose prolactin receptor. Poult. Sci. 90, $1050-1057$ (2011).

58. Liu, Z. et al. Genetic variations for egg quality of chickens at late laying period revealed by genome-wide association study. Sci. Rep. 8, 10832-10842 (2018).

59. Reddy, I. J., David, C. G. \& Singh, K. Relationship between intersequence pauses, laying persistency and concentration of prolactin during the productive period in white leghorn hens. Asian Austral. J. Anim. Sci. 18, 686-691 (2005).

60. Kulibaba, R. A. \& Podstreshnyi, A. P. Prolactin and growth hormone gene polymorphisms in chicken lines of Ukrainian selection. Cytol. Genet. 46, 390-395 (2012).

61. Reddy, I. J., David, C. G. \& Singh, K. Influence of 2-bromo- $\alpha$-ergocryptine on plasma prolactin, oestradiol-17 $\beta$ and progesterone levels in domestic hen. Asian. Austral. J. Anim. Sci. 15, 1103-1109 (2002). 
62. Ogawa, K., Matsuo, S. \& Tojo, H. Inhibitory effects of prolactin on ovulation and egg shell formation in the hen. Nihon Chikusan Gakkaiho. 48, 341-346 (1977).

63. Rozenboim, I., Tako, E., Gal-Garber, O., Proudman, J. A. \& Uni, Z. The effect of heat stress on ovarian function of laying hens. Poult. Sci. 86, 1760-1765 (2007).

64. Schwanhäusser, B. et al. Corrigendum: Global quantification of mammalian gene expression control. Nature 473, 337-342 (2011).

65. Shu, Y. \& Hong-Hui, L. Transcription, translation, degradation, and circadian clock. Biochem. Bioph. Res. Co. 321, 1-6 (2004).

66. Steckler, D., Naidoo, V., Gerber, D. \& Kähn, W. Ex vivo influence of carbetocin on equine myometrial muscles and comparison with oxytocin. Theriogenology 78, 502-509 (2012).

67. Paccamonti, D. et al. PGFM response to exogenous oxytocin and determination of the half-life of oxytocin in nonpregnant mares. Equine Vet. J. 31, 285-288 (1999).

68. Karim, M. et al. Circulating adrenomedullin does not regulate systemic blood pressure but increases plasma prolactin after intravenous infusion in humans: A pharmacokinetic study. J. Clin. Endocr. Metab. 82, 95-100 (1997).

69. Nys, Y. Relationships between age, shell quality and individual rate and duration of shell formation in domestic hens. Br. Poult. Sci. 27, 253-259 (1986).

70. Percie, D. S. N. et al. The ARRIVE guidelines 2.0: Updated guidelines for reporting animal research. PLoS Biol. 18, e3000410 (2020).

71. Chomczynski, P. \& Mackey, K. Short technical reports. Modification of the TRI reagent procedure for isolation of RNA from polysaccharide- and proteoglycan-rich sources. Biotechniques 19, 942-945 (1995).

72. Pertea, M., Kim, D., Pertea, G. M., Leek, J. T. \& Salzberg, S. L. Transcript-level expression analysis of RNA-seq experiments with HISAT, StringTie and Ballgown. Nat. Protoc. 11, 1650-1667 (2016).

73. Kim, D., Langmead, B. \& Salzberg, S. L. HISAT: A fast spliced aligner with low memory requirements. Nat. Methods. 12, 357-360 (2015).

74. Love, M. I., Huber, W. \& Anders, S. Moderated estimation of fold change and dispersion for RNA-seq data with DESeq2. Genome Biol. 15, 550 (2014).

75. Huang, D. W., Sherman, B. T. \& Lempicki, R. A. Systematic and integrative analysis of large gene lists using DAVID bioinformatics resources. Nat. Protoc. 4, 44-57 (2009).

76. Huang, D. W., Sherman, B. T. \& Lempicki, R. A. Bioinformatics enrichment tools: Paths toward the comprehensive functional analysis of large gene lists. Nucleic Acids Res. 37, 1-13 (2009).

77. Ogata, H. et al. KEGG: Kyoto Encyclopedia of Genes and Genomes. Nucleic Acids Res. 27, 29-34 (2000).

78. Ritchie, M. E. et al. Limma powers differential expression analyses for RNA-sequencing and microarray studies. Nucleic Acids Res. 43, e47 (2015).

79. Livak, K. J. \& Schmittgen, T. D. Analysis of relative gene expression data using real-time quantitative PCR and the $2^{-\Delta \Delta C T}$ method. Methods 25, 402-408 (2001).

80. Duan, Z. et al. Genetic architecture dissection by genome-wide association analysis reveals avian eggshell ultrastructure traits. Sci. Rep. 6, 28836 (2016).

\title{
Acknowledgements
}

This study was supported by the China Agriculture Research Systems (CARS-41); the National Natural Science Foundation of China (31672408); and the the Program for Changjiang Scholars and Innovative Research Team in University (IRT 15R62). We gratefully acknowledge our colleagues on the College of Animal Science and Technology of China Agricultural University for their assistance during the experiment.

\section{Author contributions}

J.X.Z., X.C., Z.X.H. and X.Z.L. developed the hypothesis and designed the overall experimental plan. X.C., Z.X.H., J.L.S., M.Y.H., X.F.S. and X.Y.L. obtained the samples and measurement data. X.C., Z.X.H. and X.Z.L. participated in data analyses. J.X.Z., G.Y.X. and J.Y.L. provide the necessary experimental resources for the development of this research. X.C., Z.X.H., G.Y.X. and J.X.Z. drafted and revised this manuscript. All authors reviewed and approved the manuscript for publication.

\section{Competing interests}

The authors declare no competing interests.

\section{Additional information}

Supplementary Information The online version contains supplementary material available at https:/doi.org/ 10.1038/s41598-021-01718-0.

Correspondence and requests for materials should be addressed to J.Z.

Reprints and permissions information is available at www.nature.com/reprints.

Publisher's note Springer Nature remains neutral with regard to jurisdictional claims in published maps and institutional affiliations.

\begin{abstract}
(c) (i) Open Access This article is licensed under a Creative Commons Attribution 4.0 International License, which permits use, sharing, adaptation, distribution and reproduction in any medium or format, as long as you give appropriate credit to the original author(s) and the source, provide a link to the Creative Commons licence, and indicate if changes were made. The images or other third party material in this article are included in the article's Creative Commons licence, unless indicated otherwise in a credit line to the material. If material is not included in the article's Creative Commons licence and your intended use is not permitted by statutory regulation or exceeds the permitted use, you will need to obtain permission directly from the copyright holder. To view a copy of this licence, visit http://creativecommons.org/licenses/by/4.0/.
\end{abstract}

(C) The Author(s) 2021 